\title{
Power consumption analysis of constant bit rate video transmission over 3G networks
}

\author{
Ukhanova, Ann; Belyaev, Evgeny; Wang, Le ; Forchhammer, Søren
}

Published in:

Computer Communications

Link to article, DOI:

10.1016/j.comcom.2012.05.010

Publication date:

2012

Document Version

Publisher's PDF, also known as Version of record

Link back to DTU Orbit

Citation (APA):

Ukhanova, A., Belyaev, E., Wang, L., \& Forchhammer, S. (2012). Power consumption analysis of constant bit rate video transmission over $3 G$ networks. Computer Communications, 35(14), 1695-1706.

https://doi.org/10.1016/j.comcom.2012.05.010

\section{General rights}

Copyright and moral rights for the publications made accessible in the public portal are retained by the authors and/or other copyright owners and it is a condition of accessing publications that users recognise and abide by the legal requirements associated with these rights.

- Users may download and print one copy of any publication from the public portal for the purpose of private study or research.

- You may not further distribute the material or use it for any profit-making activity or commercial gain

- You may freely distribute the URL identifying the publication in the public portal 


\title{
Power consumption analysis of constant bit rate video transmission over 3G networks
}

\author{
Anna Ukhanova ${ }^{\mathrm{a}, *}$, Evgeny Belyaev $^{\mathrm{b}}$, Le Wang ${ }^{\mathrm{c}}$, Søren Forchhammer ${ }^{\mathrm{a}}$ \\ ${ }^{a}$ DTU Fotonik, Technical University of Denmark, Ørsteds Plads, B. 343, 2800 Kgs. Lyngby, Denmark \\ ${ }^{\mathrm{b}}$ Tampere University of Technology, Korkeakoulunkatu 10, 33720 Tampere, Finland \\ ${ }^{c}$ Aalto University, P.O. Box 13000, FI-00076 Aalto, Finland
}

\section{A R T I C L E I N F O}

\section{Article history:}

Available online 27 May 2012

\section{Keywords:}

$3 G$

Video transmission

Constant bit rate

Power consumption modeling

Power saving

\begin{abstract}
A B S T R A C T
This paper presents an analysis of the power consumption of video data transmission with constant bit rate over 3G mobile wireless networks. The work includes the description of the radio resource control transition state machine in $3 \mathrm{G}$ networks, followed by a detailed power consumption analysis and measurements of the radio link power consumption. Based on this description and analysis, we propose our power consumption model. The power model was evaluated on a smartphone Nokia N900, which follows 3GPP Release 5 and 6 supporting HSDPA/HSUPA data bearers. We also propose a method for parameter selection for the 3GPP transition state machine that allows to decrease power consumption on a mobile device taking signaling traffic, buffer size and latency restrictions into account. Furthermore, we discuss the gain in power consumption vs. PSNR for transmitted video and show the possibility of performing power consumption management based on the requirements for the video quality.
\end{abstract}

(c) 2012 Elsevier B.V. All rights reserved.

\section{Introduction}

Wireless networks have gone through an extensive development throughout the recent years. After the Internet, in the 1990s, came the second generation wireless systems with additional features for communication by means of cellular phones. With the move from analog $(1 \mathrm{G})$ to digital transmission $(2 \mathrm{G})$, the capacity of the cellular systems have been significantly improved. Further demand for greater bandwidth resulted in the development of third generation mobile telecommunications (3G), providing a mobile broadband access to handheld devices.

The number of mobile cellular subscriptions has also been rapidly growing in the last decade, and now the rough estimate has almost reached 6 billions. Nowadays 3G systems are also becoming ubiquitous: the number of active mobile-broadband subscriptions reaches almost 1.2 billion [1]. As wireless communication technology advances, in new generation mobile phones $3 \mathrm{G}$ networks provide a large amount of various services including high data rate Internet access, videoconferencing, global positioning, high quality music and video downloading, and gaming capabilities.

Mobile TV and video-on-demand services are also rising in popularity and expected to be a significant driver for the wireless consumer industry. Consumers demand for video data, quality of

* Corresponding author. Tel.: +45 45256567, mobile: +45 50679631; fax: +45 45936581.

E-mail address: annuk@fotonik.dtu.dk (A. Ukhanova). experience of mobile multimedia and its usability is pushing the development of Mobile TV and related services. Therefore, video transmission for mobile terminals is a major application in the $3 \mathrm{G}$ and beyond systems and may play a key role in their success [2]. According to Cisco research, "two-thirds of the world's mobile data traffic will be video by 2015" [3]. As a consequence, these additional features are bringing the power consumption of mobile phones to the level of desktop computers. However, the lack of a constant power supply and limited battery capacity [4] pose strict limits to the overall power consumption of the device. Therefore, minimization of the power consumption of wireless devices is a great challenge for the entire electronic industry, at all system levels. Hence, an intense research in this field has focused on power management [5-7].

In our work we analyze the power consumption in case of uplink transmission, show how the power consumption depends on the transmission parameters and explain how it can be reduced by optimizing the power management policy. We analyze the cases with buffer and latency restrictions and show how to choose transmission parameters in these scenarios. Due to the growing interest in various mobile video applications, the analysis is then extended to the case of constant bit rate video transmission. Experimental results show that in this case the proposed solution allows to save power on video transmission if compared to the conventional approach.

Our paper starts with a review of some prior work on the power consumption in wireless networks in Section 2. Then we present 
the $3 \mathrm{G}$ mobile transmission system in Section 3, where we explain the idea of state machine applied to mobile devices and describe the communication between transmitter and receiver. In Section 4 the state power model is provided, with particular focus on Cell_FACH (Cell Forward Access Channel) and Cell_DCH (Cell Dedicated Channel) states. We propose a model of power consumption in Cell_DCH state and show that power consumption is related to the packet size and transmission interval. In Section 5 we propose a method of parameter selection for the 3GPP transition state machine that allows to decrease power consumption on the mobile device in case of constant bit rate data transmission. Section 6 presents the power consumption for constant bit rate video transmission and provides the results for trade-off between video quality and power consumption for several standard test video sequences.

\section{Power consumption in wireless networks}

There exists a large number of approaches for power management in communication networks for handheld devices and they have been studied throughout the recent years.

An overview of the methods to reduce the large and growing energy consumption of the Internet was provided in [8]. However, since that time the Internet has been adopted and widely used in many other devices other than networked desktop computers, and deployment of next generation networks in the mobile devices has significantly contributed to it.

Yeh et al. [9] as well as Sklavos and Touliou [10] provided an analysis of power consumption in 3G networks. Markov chains are used in [9] to analyze the 3GPP transition state machine and examine how different timer values affect the power consumption of the device. The analysis is based on several assumptions about the device power consumption in different states. Sklavos and Touliou [10] considered different units of the mobile phone (such as memory, display, cellular engine), analyzed power consumption on each of them and addressed different power management techniques for different units, thereby focusing on the device power consumption. They also state the need for power control techniques, e.g. based on method of idling the modules that are not used. A similar approach of dividing the processes in stages and applying different power saving mechanisms to them is described in [11]. For the reception stage, which requires most of the power, the solution is to send multimedia data in larger amounts at less frequent intervals by hiding the traffic temporarily from a mobile station, thus allowing it to sleep longer and achieve power savings. Korhonen and Wang [12] also proved that in IEEE 802.11 power of the receiver can be saved if data packets are transmitted as bursts.

Perrucci [5] provided a broad overview of existing approaches and studied the strategies for efficient use of the wireless communication in three main focus areas, namely Cross Layer, Overlay Networks and Cooperation. The energy savings on mobile devices are achieved by choosing the most efficient available network, using wake-up systems (described in detail in [13]) and sharing cellular links between mobile users. Another analysis of approaches for achieving energy-efficient web access on mobile devices is provided in [14].

Two good overviews of the past and future research directions in the field of power management for mobile networks are given in $[6,7]$. In [6] the mobile device and battery capacity evolutions, multiaccess nature of modern mobile devices and the respective implications for power management are described, as well as a proposal of an information-centric approach to networking, which allows audio/video streaming to be transformed from an energy-heavy network service to a lightweight one. However, the last would require an adoption of a new networking paradigm and a change in the way the information is distributed to its intended recipients.
Zhang et al. [7] provided a survey of many issues related to power-aware mobile multimedia, such as power-management for mobile devices, rate-distortion-complexity optimized video codec design, and computational complexity and power aware crosslayer design and optimization. The authors have pointed out the challenges and the corresponding future research directions in power-aware video coding and power-aware video delivery, such as power management in mobile devices, rate-distortion-complexity analysis of video codecs and network information feedback and cross-layer signaling.

Considering wireless video transmission from mobile devices, it is necessary to take into account that some energy has to be spent on the video capturing and compression. On average, a mobile camera may consume less than $60 \mathrm{~mW}$ for CIF resolution video at $30 \mathrm{~Hz}$ [15]. According to [16], the power needed for compression is also lower than the one needed for the transmission. Nevertheless, cross-layer approaches could be applied here as well and work like [17] discusses joint power control and bit allocation for video transmission in wireless networks. A good overview of works on this topic is also given in [18].

Chen et al. [19] described the fundamental trade-offs in wireless networks, such as deployment efficiency - energy efficiency, spectrum efficiency - energy efficiency, bandwidth - power, and delay - power trade-offs. Kim et al. [20] and Vuyst et al. [21] explored the trade-offs between delay and power consumption for sleep-mode operation in mobile WiMAX. In [22,23] the possibility for tradeoff between video quality and power saving in the receiver was demonstrated. In [22] the mobile broadcast standard DVB-H along with JPEG2000 for video encoding was used to show how to allow receivers to control the level of power consumption depending on the priorities. As any other scalable codec could be used instead of JPEG2000, the work was further extended to the Scalable Extension of H.264/AVC [23].

Unlike the work in $[22,23]$, this time we consider the case of power consumption for the uplink transmission only and thereby focus on the device. However, the main idea - that it is possible to control the trade-off between the level of power consumption and video quality - has been kept. Moreover, we also take the delay - power trade-off into account and show how it influences the achieved power savings. We provide a power model for devices for $3 \mathrm{G}$ systems, and perform power-aware cross-layer optimization by controlling the transmission parameters and adjusting the time periods spent by the mobile device in the state with active data transmission. We also show the potential for power management for video transmission that is content-dependent as different videos can have different rate-distortion characteristics.

\section{Mobile transmission system overview}

$3 G$ systems provide global communication with various services including telephony, messaging and access to Internet. $3 \mathrm{G}$ networks consist of three domains: Core Network (CN), UMTS Terrestrial Radio Access Network (UTRAN) and User Equipment (UE). UE interoperates with Base Station (called Node B). The Radio Resource Control (RRC) handles the control plane signaling between the UEs and the UTRAN. For efficient use of radio resources and power consumption control, RRC introduces a state machine for UE [24].

\subsection{State machine}

There are five states in the RRC: Idle, Cell_FACH (Cell Forward Access Channel), Cell_PCH (Cell Paging Channel), Cell_DCH (Cell Dedicated Channel) and URA_PCH (Utran Registration Area Paging Channel). Cell_PCH and URA_PCH can be considered as low power 
states, which consume only around $30 \mathrm{~mW}$. The state of Cell_FACH consumes around $400 \mathrm{~mW}$ and the state of Cell_DCH consumes around $800 \mathrm{~mW}$, according to our measurements on a smartphone Nokia N900. URA_PCH is very similar to Cell_PCH, although some vendors have not implemented it in their solution. In our work we consider these two states largely equivalent.

The power consumption in Cell_FACH is roughly $50 \%$ of that in Cell_DCH, and Cell_PCH state uses about 1-2\% of the power consumption of Cell_DCH state [25]. Each state is now described in more detail.

Idle. In this mode UE does not communicate with the network although it does listen for broadcast messages. So it does not have a RRC Connection, but UE can still have an IP address and be reached by paging. In this state the mobile device consumes the least amount of power.

Cell_PCH. In this state the channel is shared by all mobile devices so the inclusion of an additional mobile device does not have any impact on the network. UE monitors paging messages from the Radio Network Controller (RNC). As in the Idle state, the power consumption is very small. In this state no dedicated physical channel is allocated to the UE, so no uplink activity is possible.

Cell_FACH. In the Cell_FACH the mobile device communicates with the network via a shared channel. A few bits of data can be transmitted at a relatively low data rate, on the order of up to $16 \mathrm{kbps}$ in the uplink. The maximum amount of transmission data also depends on the overall loading of the common channels. At the same time the UE continuously monitors a FACH in the downlink. The mobile device power consumption is higher than it is in Idle or Cell_PCH states.

Cell_DCH. The mobile device is allocated a dedicated transport channel both in downlink and uplink. It consumes the most network resources and the impact on the battery is at the very high level.

\subsection{Communication between transmitter and receiver}

It is UE that always initiates the RRC connection, then the establishment and the release are handled by the RRC protocol. UE starts working in Idle state, when an RRC connection has not yet been established. Only one RRC connection is used at any time between the UE and the network. When an RRC connection has been established between UE and Node B together with RNC, the Idle state switches to the RRC Connected mode.

To be more precise, from Idle mode through establishment of an RRC connection the UE enters the Cell_DCH state. Further it can be moved by explicit signaling from Cell_DCH to other states. The UE does not generally listen to the broadcast channel in this state. If Node B allocates to UE a common or shared traffic channel (i.e., the channel is shared by several UEs), it enters Cell_FACH state. The data communication activities can only be performed in these two states.

Depending on the activities of the UE and traffic volume, states could be changed. Signaling messages (radio bearer configuration messages) are sent between UE and Node B when states are changing. Three timers are used to detect when a mobile device should move to a lower power state in case of inactivity. These inactivity timers T1, T2, and T3 are managed by RNC.

T1 is used in Cell_PCH. After T1 seconds (usually a very long timer), the RRC connection will be released and the state will be changed to Idle. T2 is an inactivity timer determining how long the 3G device should remain in Cell_FACH state without any activity. Timer T3 is used within Cell_DCH state and refers to the inactivity period after which the 3G device enters Cell_FACH.

As for the signaling traffic i.e. messages between UE and Node B, it is necessary to note that $3 \mathrm{G}$ was designed and implemented to support large amounts of data traffic (like long, uninterrupted data sessions, video conferencing, etc.). But according to Thelander, CEO and founder of Signals Research Group (SRG), the reality is that "signaling traffic is outpacing actual mobile data traffic by $30-$ $50 \%$, if not higher" [26].

\section{Power consumption analysis of transition states}

In this section, the power consumption of each state of the RRC is analyzed. We focus on modeling the power consumption in Cell_DCH state. Then, our proposed model is compared with real measurements and a reference model which thoroughly analyzes power consumption for UDP traffic in 3G network and provides practical power model for the consumption.

\subsection{The influence of packet sending intervals and packet size on power consumption of Cell_DCH state}

Each RRC state requires varying power to maintain operation and differs when generating signals transferred between UE and RNC to establish, maintain and release connections as well as transmit/receive data across the air interface. Compared to Cell_FACH state, which is only applicable for transferring relatively small quantities of data, Cell_DCH state gives potential for UE to transfer large quantities of data and, thus, is the state where most of the data communication happens, as specifically described in the previous section. It is the most interesting state to examine the effect of packet sending intervals and packet size on power consumption of UE radio interface.

Generally speaking, the size of a transport block specifies the maximum payload that can be transmitted within each Transmission Time Interval (TTI), which decides the maximum packet sending or receiving rate. These two parameters together influence the maximum throughput and packet sending or receiving pattern in the Physical layer. From the Transport layer perspective, application's traffic pattern - namely packet sending or receiving interval and packet size - directly decides underlaying layer's behavior regarding the size of transport block set and transmitting interval. Specifically, if the number of bits in a TTI is larger than the maximum size which one physical block can contain, segmentation is performed and the over-sized bits are sent during the next TTI. If the packets are generated with an interval which is less than TTI, the transmitting interval is decided by TTI. Otherwise, it is decided by the packet generating interval of the application. The power consumption of a radio interface increases proportionally to the number of transport block sets sent and received over one radio interface.

Fig. 1 demonstrates the power consumption of packet transmission on a smartphone Nokia N900 in downlink. A traffic generator is used to generate UDP packets in different intervals as shown in the figure. Each peak corresponds to the power consumption of transmitting one transport block set. As it can be seen, the number of peaks directly influences the power consumption of the radio interface. A faster receiving interval leads to a larger number of peaks, and thus higher power consumption. In our experiments, we also observed the same phenomena in uplink on Nokia N900. Among the peaks, wide ones are due to the power consumption of daemon processes of $3 \mathrm{G}$ modem and excluded from calculation in the following subsections. The power consumption was measured in a stable environment. Otherwise, when the signal quality of UE drops off or the UE moves away from the Node B, power control mechanisms in 3G network have to increase transmission power in order to keep the received uplink Signal-to-Interference Ratio (SIR) at a given SIR target. Moreover, the degradation of signal quality also increases MAC layer retransmission, which leads to more energy consumption for successful transmission of one bit. 

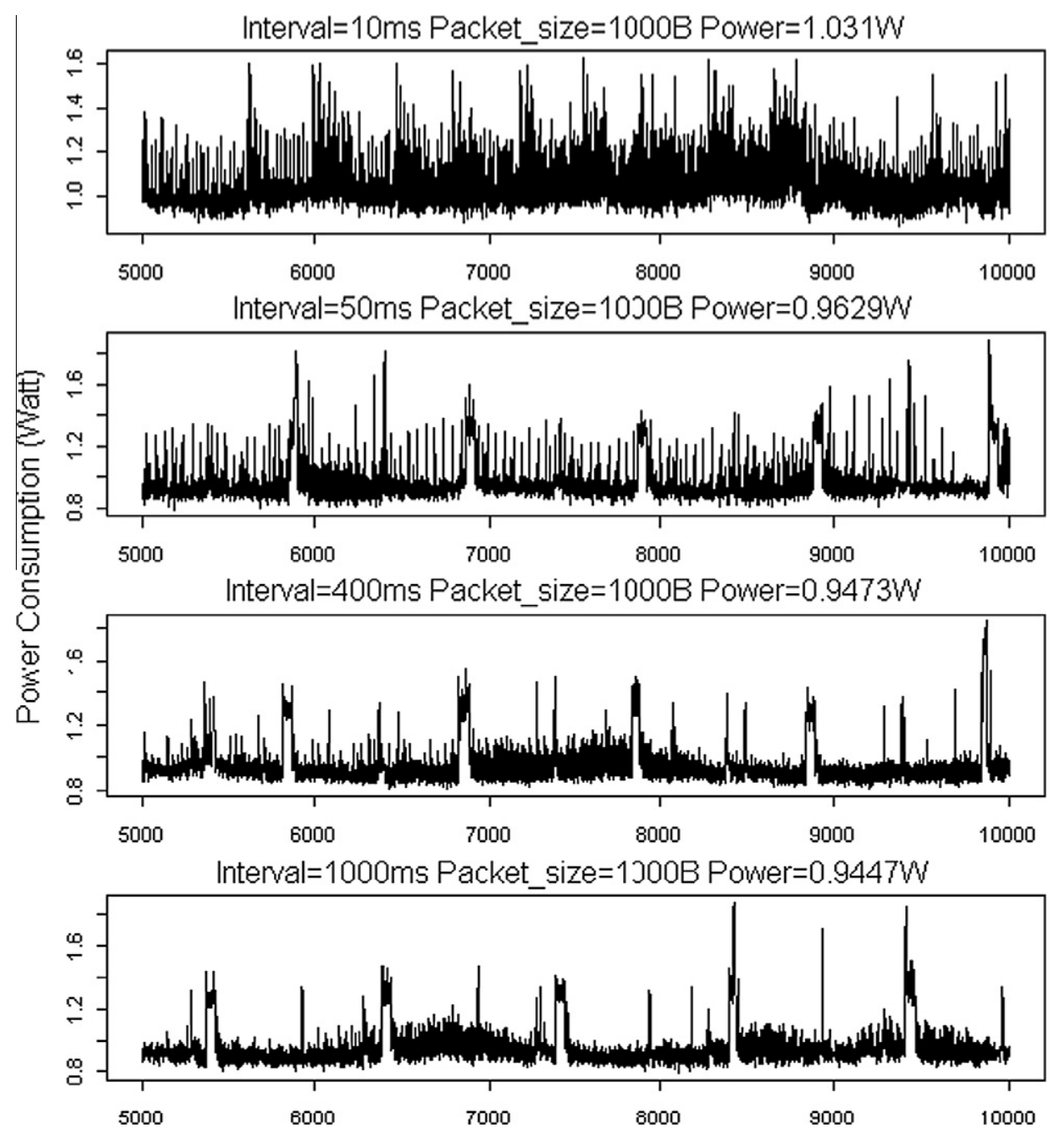

Fig. 1. Power consumption of Nokia N900 in downlink.

The measurements were done three times. The average variance of the transmission power consumption (sending and receiving) is $5.7 \cdot 10^{-6}$.

\subsection{Power consumption modeling of Cell_DCH state}

The power consumption of sending or receiving packets in Cell_DCH state mainly consists of three sources: the power consumption of Cell_DCH state defined as $P_{D C H}$ in watt, the power consumption of sending or receiving packets which is defined as $P_{\text {peak }}$ in watt demonstrated as power peaks in Fig. 1, and the power consumption of encapsulating or decapsulating packets which is defined as a function of packet size $P_{\text {enc }}(s)$, where $s$ is the size of packet in bytes. Therefore, the power consumption of UE to send or receive packets in state Cell_DCH can be reformulated as follows:

$P=P_{D C H}+P_{\text {peak }}+P_{\text {enc }}(s)$.

UE requires resource and power consumption to maintain the state. Thus, $P_{D C H}$ is the minimal power consumption for the UE to stay in state Cell_DCH, which includes the power consumption of reception of control signals and is considered as an approximately fixed value since most of the traffic is data traffic.

Besides, we define $I$ as the UDP packet sending interval in Transport layer in ms, which is the interval of sending packets from UE to Node B or the interval of sending packets from Node B to UE.
The power consumption for encapsulation or decapsulation $P_{\text {enc }}(s)$ for packet size $s$ is assumed to be linear and proportional to the size of the packet since more computational power is required to process bigger packets yielding higher power consumption. Since $P_{D C H}, P_{\text {peak }}$ and total power consumption $P$ are measurable, the power component $P_{\text {enc }}(S)$ can be calculated and then used to derive the linear model.

The number of transport blocks needed for sending one IP packet is determined by the size of the packet and maximum number of bits for a transport block per TTI defined in different 3GPP releases. Here we define the number of transport blocks as

$N=\left\lceil\frac{S}{M T B S}\right\rceil$,

where MTBS is Maximum Transport Block Size.

Let $E_{\text {peak }}$ denote the energy consumption of sending or receiving one peak in Joule. As analyzed above, the power consumption of peaks changes more or less linearly with the number of the transport blocks. When more than one transport block is needed to send or receive one IP packet, the time spent on processing this packet is $N \cdot \tau$, where $\tau$ is the value of TTI. Normally, packet sending interval $I$ is much larger than the packet processing time. Thus,

$P_{\text {peak }}=\frac{N}{I} \cdot E_{\text {peak }}, \quad$ when $I \gg N \cdot \tau$.

Then combining (1)-(3), the power consumption in Cell_DCH state can be written as 
$P=P_{D C H}+\frac{E_{\text {peak }}}{I}\left(\left\lceil\frac{s}{M T B S}\right\rceil\right)+P_{\text {enc }}(s)$.

Eq. (4) formulates the power consumption of one connection of uplink or downlink traffic. This model shows that power consumption can be determined by the number of peaks, namely set by packet sending or receiving interval $I$ and packet size $s$, which is the influential factor of power consumption of the radio interface. This model can be extended to formulate the power consumption for multiple connections by counting the amount of bits sent or received in a certain interval. As long as mobile devices are capable to record transmitted and received packet intervals and sizes, the proposed model can be extended to estimate power consumption of the radio interface in runtime.

\subsection{Experimental setup}

In order to have sufficient measurement accuracy and an uninterrupted power source, the battery of the N900 was replaced with a battery adapter, which was serially connected to a $4.1 \mathrm{~V} \mathrm{DC}$ power supply and a $0.1 \mathrm{Ohm}$ resistor. A NI cRIO-9215 was then used as a data logger to record voltage fluctuations of the N900 at a sample rate of 1000 sample/s. A Linux traffic generator was also used to generate packets with various packet sizes and sending intervals. UDP traffic was generated instead of TCP to avoid TCP hand-shake and retransmissions. The packet sending interval ranged from $10 \mathrm{~ms}$ to $1000 \mathrm{~ms}$ to avoid UE switching to Cell_FACH state. The packet size ranged from 10 bytes to 1500 bytes, which is the typical Maximum Transmission Unit (MTU) for Ethernet. When measuring the power consumption of sending packets, the UDP packets generated on the N900 were sent to Node B via uplink, and then forwarded to the Linux server. When measuring the power consumption of receiving packets, the UDP packets were generated on the Linux server and received by the N900 in downlink.

\subsection{Evaluation of power models}

The power model was evaluated on Nokia N900, which follows a 3GPP Release 5 and 6 supporting HSDPA/HSUPA data bearers. The measured average energy consumption of peak $E_{\text {peak }}$, average power consumption in Cell_DCH $P_{D C H}$ and related parameters needed for (1) are listed in Table 1 . Through (4), the power consumption of sending or receiving packets is thereby decided by $I$ and $s$.

In order to evaluate our model, we use another power consumption model, which is based on the assumption that power consumption is linear in the data rate $r$ [27]. The referenced model designates two independent variables.

- Time of data communication.

- Amount of data sent or received during the communication.

Table 1

Parameters for the experiment applied on Nokia N900.

\begin{tabular}{lll}
\hline & Uplink & Downlink \\
\hline UE category & HSUPA category 5 & HSDPA category 5 \\
TTI & $10 \mathrm{~ms}$ & $2 \mathrm{~ms}$ \\
Maximum Transport & $20000 \mathrm{bits}$ & $7298 \mathrm{bits}$ \\
$\quad$ Block Size (MTBS) & & \\
Data rate & $2 \mathrm{Mbit} / \mathrm{s}^{\mathrm{a}}$ & $2 \mathrm{Mbit} / \mathrm{s}$ \\
$E_{\text {peak }}$ & $0.4532 \mathrm{E}-3 \mathrm{~J}$ & $0.4435 \mathrm{E}-3 \mathrm{~J}$ \\
$P_{D C H}$ & $0.8556 \mathrm{~W}$ & $0.8478 \mathrm{~W}$ \\
\hline
\end{tabular}

a Note: The uplink data rate is limited to $2 \mathrm{Mbit} / \mathrm{s}$ due to the type of data packet. The maximum data rate is $3.65 \mathrm{Mbit} / \mathrm{s}$.
The energy consumption of UDP-type session, therefore, is expressed as follows.

$E=t \cdot\left(r_{t}+r \cdot r_{d}\right)+c$,

where $r_{d}$ is the energy consumption rate for data in Joule/KByte, $r_{t}$ is power consumption in Watt, $r$ is data rate, $t$ is transmission time in second and constant $c$ represents the offset term of the energy consumption, which is independent of the process duration. The model is introduced as a general equation of energy consumption to describe UDP-type of data session. In order to compare it with our power consumption model, the equation needs to be divided by time $t$. The power consumption model can be formulated as a linear equation.

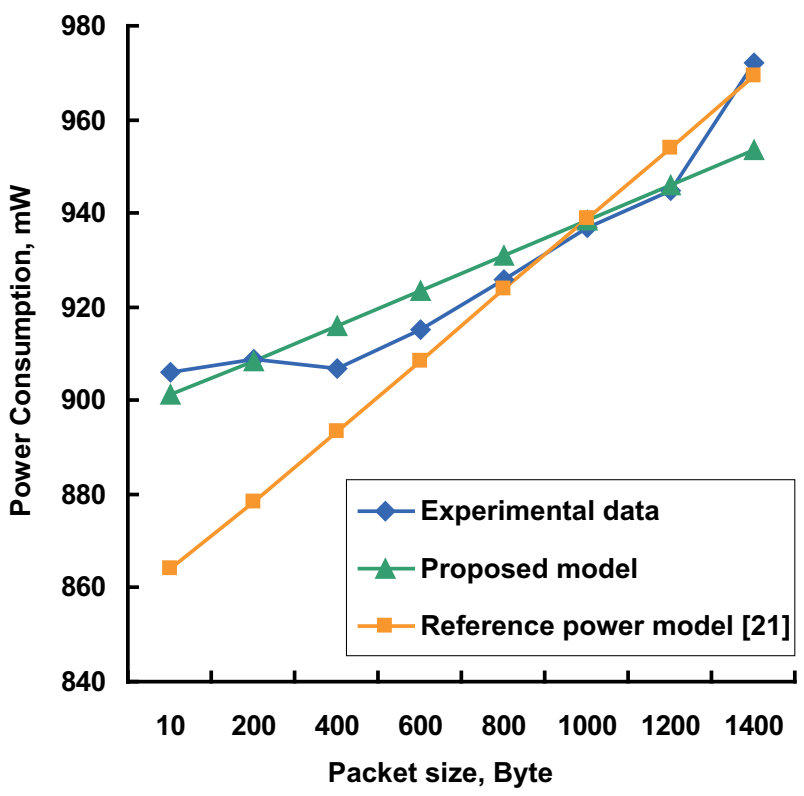

Fig. 2. Uplink power consumption for experiment, proposed model and reference model for time interval $=10 \mathrm{~ms}$.

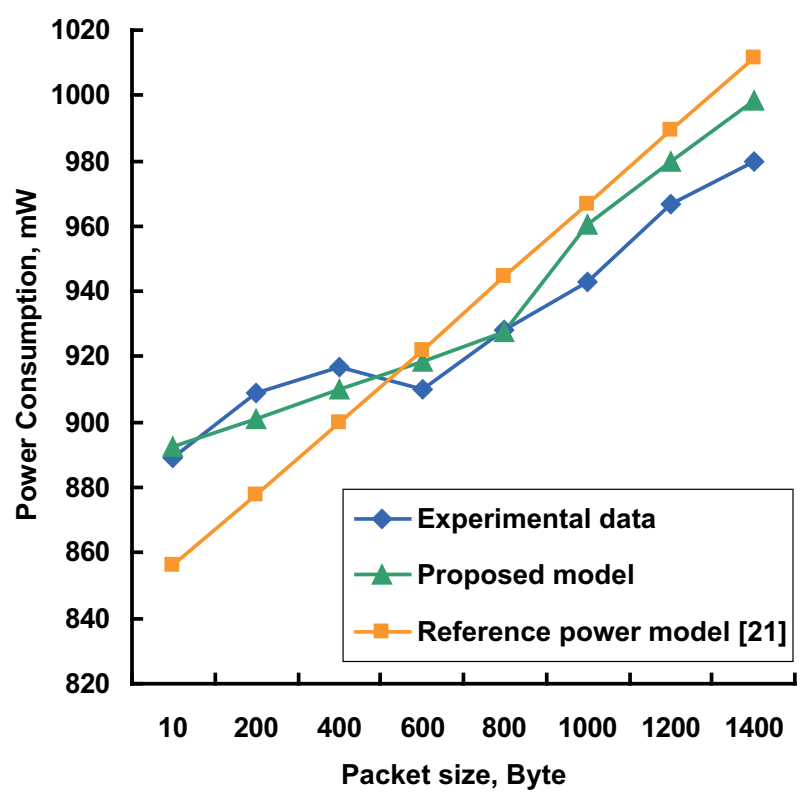

Fig. 3. Downlink power consumption for experiment, proposed model and reference model for time interval $=10 \mathrm{~ms}$. 
$P=r_{t}+r \cdot r_{d}$

The fitted values of the proposed model and reference model, and the measured values from experiments are shown in Figs. 2 and 3. To compare two models, rate $r$ in the reference model is expressed as the product of packet size $s$ and packet sending frequency $I^{-1}$ such that two models share the same coordinate. Our proposed model shows a better approximation than the reference model. We also compared the two models with their Mean Absolute Percentage Error (MAPE) for downlink and uplink power consumption, which is defined as follows.

$M A P E=\frac{1}{n} \cdot \sum_{i=0}^{n} \frac{\left|A_{i}-F_{i}\right|}{A_{i}}$,

where $n$ is the number of measured values of power consumption, $A_{i}$ is the actual value and $F_{i}$ is the fitted value. The MAPEs of the proposed model is $5.0 \%$ for downlink and $4.7 \%$ for uplink power consumption, which is about $28 \%$ more accurate than that of the reference model.

\section{Uplink power consumption analysis of transition state machine}

Section 5 provides the uplink power consumption analysis of the transition state machine (TSM) described in Section 3. As we consider constant bit rate sources (in contrast to http-like data sources, modeled e.g. as Poisson source traffic [28]), it is easy to predict the source rate and find optimal parameters for TSM to minimize the uplink power consumption. This section starts with an evaluation of power consumption for ideal transition state machine. Further this ideal TSM is used for parameter selection for 3GPP TSM, and the differences between these two TSMs are also described. The paper continues with the analysis of the TSM and parameter selection in case for different limitations: the limitation for the amount of signaling traffic (Section 5.3), the limitations for the amount of signaling traffic and buffer size (Section 5.4), and the limitations for the amount of signaling traffic and buffering latency (Section 5.5). The corresponding results for power savings in these cases are presented on the figures in this Section.

\subsection{Power consumption for ideal transition state machine}

Let $P$ denote the uplink power consumption of mobile device, $r$ the data source bit rate and $c$ the channel bit rate. The optimal TSM has to correspond to the following optimization task:

$\left\{\begin{array}{l}\text { minimize } P, \\ c \geqslant r .\end{array}\right.$

Let us define $B_{R L C}$ as the uplink data buffer of UE, $B_{R L C}^{T}$ as the buffer threshold and $t_{\text {inact }}$ as the inactivity time. Then, the following ideal TSM can be introduced:

State 1 (Cell_PCH).

If activity detection, then go to State 2 ,

else go to State 1 .

State 2 (Cell_FACH).

If $B_{R L C}>B_{R L C}^{T}$, then go to State 3,

else if $t_{\text {inact }}>T_{2}$, then go to State 1 ,

else go to State 2 .

State 3 (Cell_DCH).

If $B_{R L C}=0$, then go to State 2,

else go to State 3 .

Let us define $p_{1}, p_{2}$ and $p_{3}$ as the power consumption in State 1 , State 2 and State 3 respectively and $c_{2}$ and $c_{3}$ as channel rate in
State 2 and State 3 . The power consumption of this TSM depending on the data source bit rate $r$ can be described as follows. If data rate $r=0$, then UE is always working in State 1 and power consumption is $p_{1}$. If $0<r \leqslant c_{2}$, then UE is always working in State 2 and has power consumption $p_{2}$. If $r \geqslant c_{3}$, then UE is always working in State 3 and has power consumption $p_{3}$. If $c_{2}<r<c_{3}$ then there is buffer accumulation in State 2 and buffer emptying in State 3. Accumulation time in State 2 is

$t_{2}=\frac{B_{R L C}^{T}}{r-c_{2}}$.

Emptying time in State 3 is

$t_{3}=\frac{B_{R L C}^{T}}{c_{3}-r}$.

Finally, power consumption for the ideal TSM is:

$P= \begin{cases}p_{1}, & \text { if } r=0, \\ p_{2}, & \text { if } 0<r \leqslant c_{2}, \\ \frac{t_{2}}{t_{2}+t_{3}} \cdot p_{2}+\frac{t_{3}}{t_{2}+t_{3}} \cdot p_{3}, & \text { if } c_{2}<r<c_{3}, \\ p_{3}, & \text { otherwise. }\end{cases}$

Theorem 1. The state machine described above is a solution of the optimization task (8) for data source bit rate $r \in\left(c_{2}, c_{3}\right)$.

Proof. From (11) it follows that for ideal TSM the channel rate $c=r$ for $r \in\left(c_{2}, c_{3}\right)$. Let us assume that another TSM with power consumption $P^{\prime}<P$ exists. It is possible only if accumulation time $t_{2}^{\prime}$ in State 2 for this TSM is more than the accumulation time $t_{2}$ in State 2 for ideal TSM. But in this case channel rate $c^{\prime}$ for this TSM will be less than $r$. It means that this TSM does not exist.

\subsection{Power consumption for the 3GPP transition state machine}

The ideal TSM can be used to select the parameters for 3GPP TSM [29]: $B_{R L C}^{T}$ which was defined in previous subsection and timer $T_{3}$. Then 3GPP TSM differs from the ideal TSM only in State 3, that can be described as follows (see Fig. 4):

State 3 (Cell_DCH).

If $r<c_{3}$ more than $T_{3} \mathrm{sec}$, then go to State 2,

else go to State 3 .

If data rate $r=0$, then UE is always working in State 1 and power consumption is $p_{1}$. If $0<r \leqslant c_{2}$, then UE is always working in State 2 and has power consumption $p_{2}$. If $r \geqslant c_{3}$, then UE is always working in State 3 and has power consumption $p_{3}$. If $c_{2}<r<c_{3}$ then there is buffer accumulation in State 2 and buffer emptying in State 3. Finally, a power consumption model for 3GPP TSM is given by (11) with accumulation time in State 2

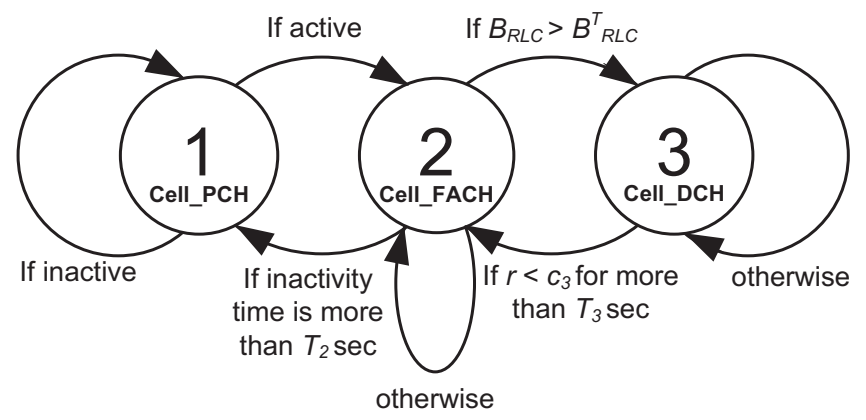

Fig. 4. 3GPP transition state machine. 
$t_{2}=\frac{\min \left\{B_{R L C}^{T},\left(c_{3}-r\right) \cdot T_{3}\right\}}{r-c_{2}}$

and emptying time in State $3 t_{3}=T_{3}$.

From Theorem 1 follows that if parameters $T_{3}$ and $B_{R L C}^{T}$ of $3 G P P$ TSM satisfy the equation

$B_{R L C}^{T}=\left(c_{3}-r\right) \cdot T_{3}$,

then 3GPP TSM is identical to ideal TSM and it is a solution of the optimization task (8) too.

Figs. 5 and 6 show power consumption for TSM with experimentally obtained parameters and parameters for carrier 1 listed

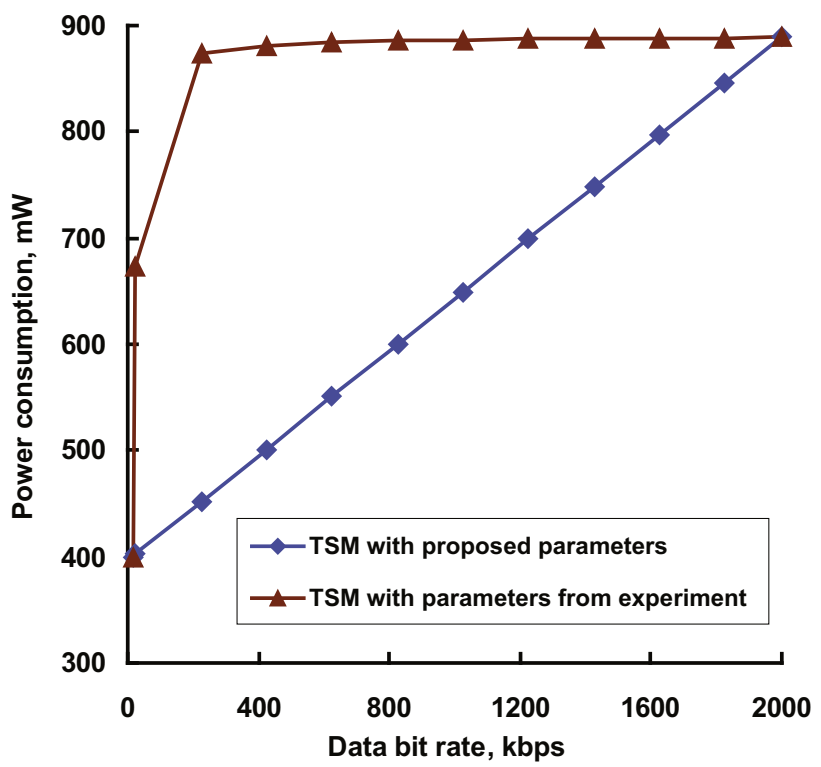

Fig. 5. Uplink power consumption for transition state machine for constant bit rate data transmission, $c_{2}=16 \mathrm{kbps}, c_{3}=2000 \mathrm{kbps}, B_{R L C}=8 \mathrm{kB}, T 3=9 \mathrm{~s}, p_{2}=400$ $\mathrm{mW}, p_{3}=890 \mathrm{~mW}$.

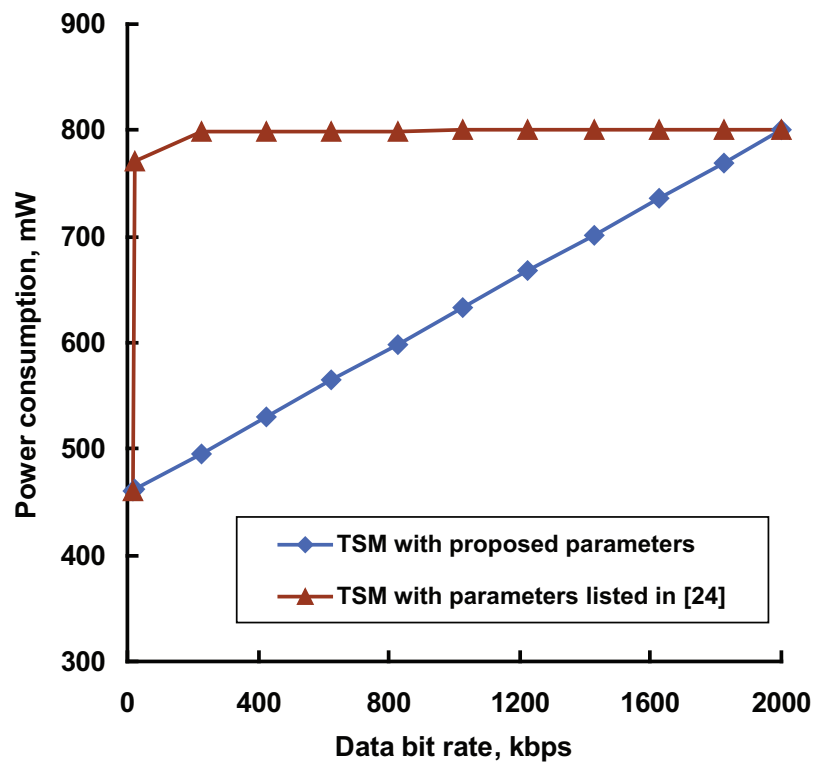

Fig. 6. Uplink power consumption for transition state machine for constant bit rate data transmission, $\quad c_{2}=16 \mathrm{kbps}, \quad c_{3}=2000 \mathrm{kbps}, \quad B_{R L C}=543 \mathrm{~B}, \quad T 3=5 \mathrm{~s}$, $p_{2}=460 \mathrm{~mW}, p_{3}=800 \mathrm{~mW}$. in [24] and compared with TSM with proposed parameters selected using (13) for constant bit rate data. In fact, the parameters depend on the operator; some additional data and discussions could be found in [30]). These figures illustrate that with the proposed parameter selection the power consumption is growing linearly with the growth of bit rate, which allows a significant decrease of uplink power consumption of UE.

\subsection{Parameter selection taking signaling traffic into account}

As was mentioned in Section 3, one of the problems for 3G networks is the increasing amount of the signaling traffic $[31,32,24]$, as each time UE moves between the various RRC states it exchanges signaling messages with the mobile network for mobility and radio resource management. In this subsection we show the possibility of controlling the total signaling traffic on the base station.

Let us define $n_{23}$ as the number of signaling messages needed to be transmitted from UE to base station for the transition from State 2 to State 3 and $n_{32}$ as the one needed for the transition from State 3 to State 2 and $n_{s}$ as the number of signaling messages per second for UE. Then, optimization task (8) can be modified as follows:

$$
\left\{\begin{array}{l}
\text { minimize } P, \\
c \geqslant r, \\
n_{s} \leqslant N_{s}^{*},
\end{array}\right.
$$

where $N_{s}^{*}>0$ is a maximum allowed number of signaling messages per second for UE.

If $c_{2}<r<c_{3}$ the UE works in State 2 and State 3 only. In case of constant bit rate transmission all working time can be divided into the equal intervals $t_{2}+t_{3}$ seconds (see Fig. 7). The number of transmitted signaling messages in each interval is a sum of transition signaling messages for both states $n_{23}+n_{32}$. Therefore the number of signaling messages per second can be calculated as

$n_{s}=\frac{n_{23}+n_{32}}{t_{2}+t_{3}} \leqslant N_{s}^{*}$.

Combining (9), (10) and (15), it follows:

$B_{R L C}^{T} \geqslant \frac{\left(n_{23}+n_{32}\right) \cdot\left(r-c_{2}\right) \cdot\left(c_{3}-r\right)}{N_{s}^{*} \cdot\left(c_{3}-c_{2}\right)}$.

The maximum needed $B_{R L C}^{T}$ is

$\max _{r} B_{R L C}^{T}=\frac{\left(n_{23}+n_{32}\right) \cdot\left(c_{3}-c_{2}\right)}{4 \cdot N_{s}^{*}}$.

From (17) follows that 3GPP TSM is a solution of (14) if

$\left\{\begin{array}{l}B_{R L C}^{T}=\frac{\left(n_{23}+n_{32}\right) \cdot\left(c_{3}-c_{2}\right)}{4 \cdot N_{s}^{*}}, \\ T_{3}=\frac{B_{R L C}^{T}}{c_{3}-r} .\end{array}\right.$

In case of constant bit rate data transmission, (18) allows to control the total network signaling traffic on the base station by selecting $N_{s}^{*}$ for each UE.

\subsection{Parameter selection taking signaling traffic and UE buffer size restrictions into account}

In real applications the UE buffer size can be limited depending on device properties. In this case, it is important to comply with this limitation while choosing the parameters for the transmission in the proposed method. Taking into account buffer size restrictions, the optimization task (14) can be modified as follows: 


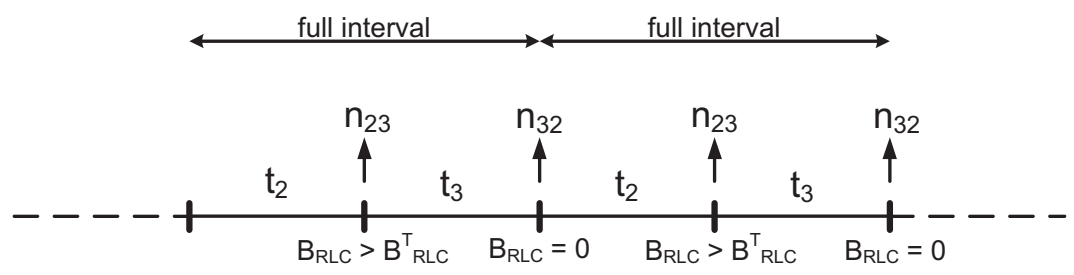

Fig. 7. Amount of signaling traffic for a transmission interval.

$\left\{\begin{array}{l}\text { minimize } P, \\ c \geqslant r, \\ n_{s} \leqslant N_{s}^{*}, \\ B_{R L C}^{T} \leqslant B_{R L C}^{\max },\end{array}\right.$

where $B_{R L C}^{\max }$ is the maximum possible UE buffer size.

Taking into account buffer size restrictions, the working time of UE in State 2 is

$t_{2}=\frac{B_{R L C}^{\max }}{r-c_{2}}$.

At the same time the working time of UE in State 3 can be written as

$t_{3}=\frac{B_{R L C}^{\max }}{c_{3}-r}+\Delta t_{3}$

where $\Delta t_{3} \geqslant 0$ is an additional time needed to provide the maximum allowed number of signaling messages per second $N_{s}^{*}$.

Taking into account (15), (20) and (21), the additional time has to satisfy the following inequality:

$\Delta t_{3} \geqslant \frac{n_{32}+n_{23}}{N_{s}^{*}}-\frac{B_{R L C}^{\max }}{r-c_{2}}-\frac{B_{R L C}^{\max }}{c_{3}-r}$.

To minimize the power consumption and provide $c=r$ the inequality in (22) should be written as equality. As a result, for $c_{2}<r<c_{3}$ 3GPP TSM will be a solution of (19) if

$\left\{\begin{array}{l}B_{R L C}^{T}=B_{R L C}^{\max }, \\ T_{3}=\frac{B_{R L C}^{\max }}{c_{3}-r}+\max \left\{0, \frac{n_{32}+n_{23}}{N_{s}^{*}}-\frac{B_{R L C}^{\max }}{r-c_{2}}-\frac{B_{R L C}^{\max }}{c_{3}-r}\right\} .\end{array}\right.$

Figs. 8 and 9 show the comparison between the power consumption for systems with TSM without any restrictions and with different buffer limitations. Formula (23) shows that with the increase of restricted $N_{s}^{*}$ the time portion of working in State 2 becomes closer to the maximum possible value without restrictions (18). For low values of $N_{s}^{*}$ there is an opposite situation, and the UE should stay longer in State 3, therefore the gain in power consumption savings is lower. For $N_{s}^{*}=2$ only the curve for $B_{R L C}^{\max }=$ $200 \mathrm{kB}$ differs from the one without restrictions. That means the value of $N_{s}^{*}$ is high enough to adapt the parameter values, unless the buffer becomes too small to keep the UE in State 2 long enough. Thus, the result of the gain in power consumption savings with buffer restrictions depends on the current situation in the network and limitations on $N_{s}^{*}$, imposed by the network operator.

5.5. Parameter selection taking signaling traffic and buffering latency restrictions into account

Another important restriction is buffering latency $\Delta T$, which has a significant meaning for real-time wireless communications. Necessary requirement for conversational services is an end-to-end delay smaller than $250 \mathrm{~ms}$ [33], but, according to [34], the desired delay in real time video applications is approximately $40 \mathrm{~ms}$. For non-real time video applications the limit value is higher and should not be greater than $10 \mathrm{~s}$, as stated in [35]. This limitation

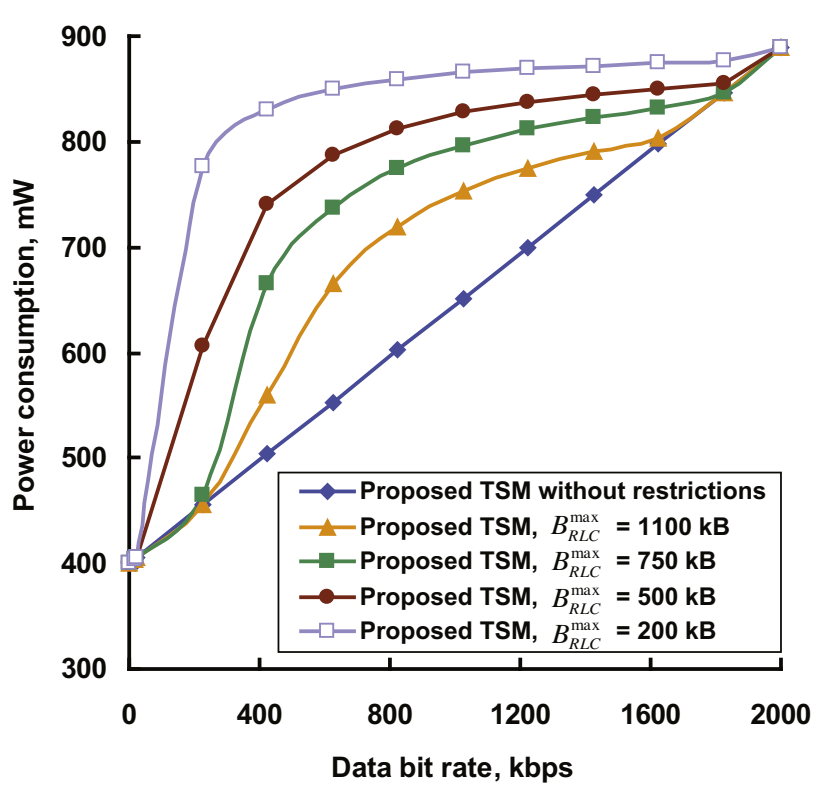

Fig. 8. Power consumption with buffer restrictions and $N_{s}^{*}=0.4$.

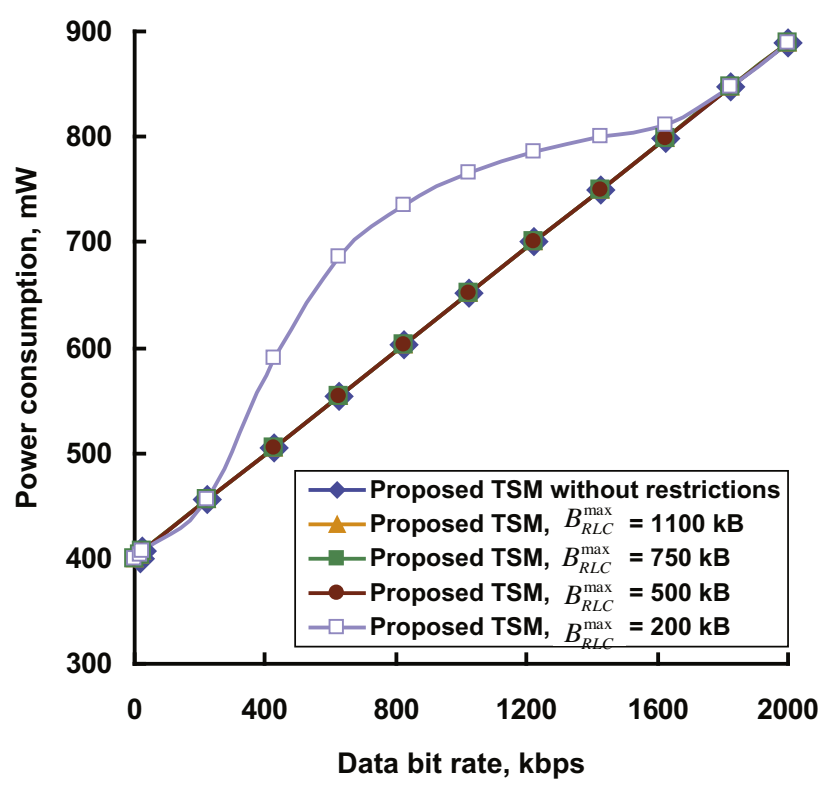

Fig. 9. Power consumption with buffer restrictions and $N_{s}^{*}=2$.

for buffering latency also influences our method for efficient power savings.

Taking buffering latency restriction into account, the optimization task (14) can be modified as follows: 
$\left\{\begin{array}{l}\text { minimize } P, \\ c \geqslant r, \\ n_{s} \leqslant N_{s}^{*}, \\ \Delta T \leqslant L,\end{array}\right.$

where $L$ is a buffering latency restriction.

Taking this restriction into account, the working time of UE in State 2 is

$t_{2}=L$.

At the same time the working time of UE in State 3 can be written as

$t_{3}=\frac{L \cdot\left(r-c_{2}\right)}{c_{3}-r}+\Delta t_{3}$,

where $\Delta t_{3} \geqslant 0$ is an additional time needed to provide the maximum allowed number of signaling messages per second $N_{s}^{*}$. Taking (15) into account, $\Delta t_{3}$ has to satisfy the following inequality:

$\Delta t_{3} \geqslant \frac{n_{32}+n_{23}}{N_{s}^{*}}-L-\frac{L \cdot\left(r-c_{2}\right)}{c_{3}-r}$.

In order to minimize the power consumption and provide $c=r$, the inequality in (27) should be written as equality. As a result, for $c_{2}<r<c_{3}$ 3GPP TSM will be a solution of (24) if

$\left\{\begin{array}{l}B_{R L C}^{T}=L \cdot\left(r-c_{2}\right), \\ T_{3}=\frac{B_{R L C}^{T}}{c_{3}-r}+\max \left\{0, \frac{n_{32}+n_{23}}{N_{s}^{*}}-L-\frac{L \cdot\left(r-c_{2}\right)}{c_{3}-r}\right\} .\end{array}\right.$

Figs. 10 and 11 show the comparison between the power consumption for system with TSM without any restrictions and TSM with different latency limitations. As in the case with buffer limitations, (28) shows that with the increase of $N_{s}^{*}$, the time portion of working in State 2 can come closer to the maximum possible value without restrictions, defined by (18). Instead, low values of $N_{s}^{*}$ mean that the UE will work more in State 3, and there is a smaller gain in power consumption savings compared to the case without any restrictions. For $N_{s}^{*}=2$ a significant gain can be obtained for latencies bigger than $5 \mathrm{~s}$. On one hand, this is caused by the fact that limitation on latency allows for UE to stay in State 2 long enough to achieve the decrease of power consumption. At the same time, due to a reasonably high value of $N_{s}^{*}$, the UE can also move to State 3 and come back to State 2 after transmission often en-

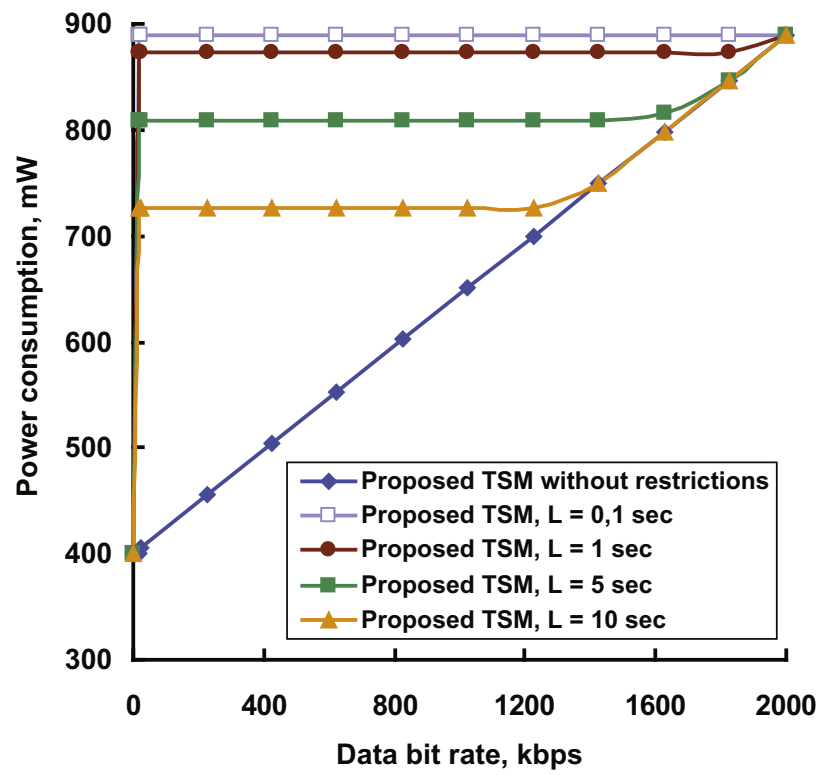

Fig. 10. Power consumption with latency restrictions and $N_{s}^{*}=0.4$.

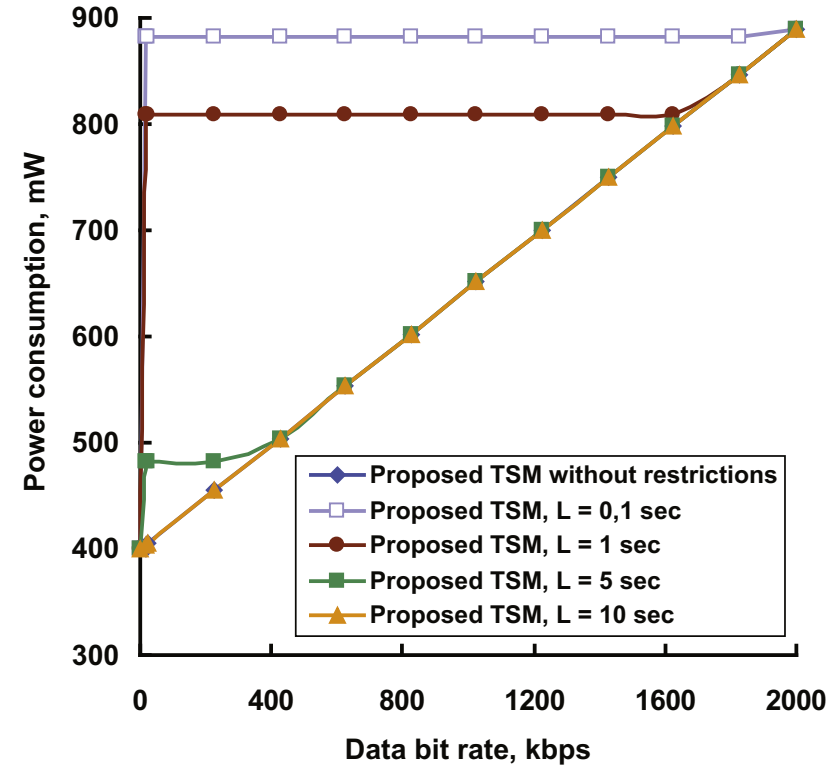

Fig. 11. Power consumption with latency restrictions and $N_{s}^{*}=2$.

ough. So, generally speaking for the case with latency restrictions, as well as for the case with buffer limitations, the resulting gain in power consumption savings depends on the limitations on $N_{s}^{*}$.

\section{Energy efficient constant bit rate video transmission over 3GPP networks}

In this section the analysis of video transmission over 3G networks is presented. We are focusing our research on power consumption of constant bit rate video only. One source of constant bit rate video data is a single-layer codec with rate control mechanism that helps to achieve constant bit rate of the compressed video data [36,37]. Constant bit rate transmission of variable bit rate stored video may be performed e.g. with the optimal choice of buffer size [38].

The system in general can operate as described on the Fig. 12. The UE decides the power saving mode according to its needs, and based on it the power saving controller chooses the optimal video bit rate, as demonstrated later in this section. At the same time, power saving controller finds the optimal parameters for RRC state machine, as described in Section 5. Based on the given video bit rate, the video rate controller defines the compression parameters, which are used for encoding the video source. After this the compressed bit stream is ready for the transmission with optimal TSM parameters, providing the necessary level of power consumption for the UE.

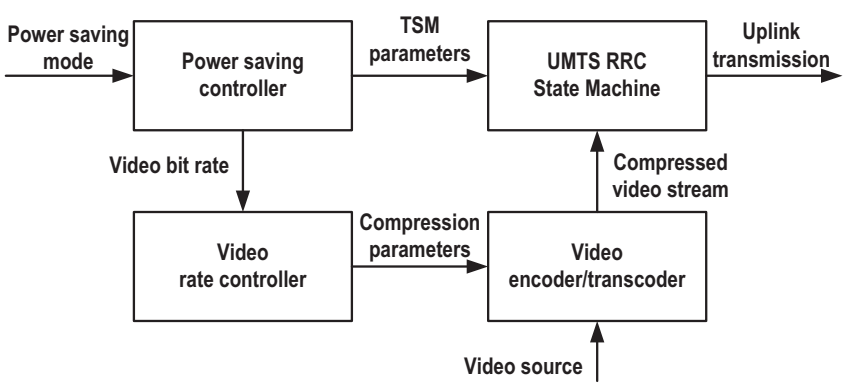

Fig. 12. UE power saving based on trade off between video quality and power consumption. 
Another approach considers the use of scalable video coding (e.g. Scalable Extension of H.264/AVC [39]), with further extraction of the necessary amount of data under bit rate constraints (at the same time, possibly maximizing quality) [40]. The latter approach can practically be used in hybrid video surveillance systems, consisting of a camera that records video data in a scalable format and a transmission terminal that is used for the delivery of the encoded video data. For power saving reasons, in case of no motion detection, the camera can transmit the base layer only. When the motion is detected, the camera starts transmitting several (up to all) quality layers of the video sequence, thus increasing the power consumption of the mobile terminal.

In this section we evaluate the necessary amount of power for the video transmission for different levels of video quality. For practical experiments the H.264/AVC scheme in reference software JM codec v.16.2 [41] was used. Experimental results were obtained for several test video sequences: "Hall monitor", "Foreman", "Soccer" and "Akiyo" at QCIF $(176 \times 144)$ resolution, $15 \mathrm{~Hz}, 300$ frames and CIF $(352 \times 288)$ resolution, $30 \mathrm{~Hz}, 300$ frames, and also "Crew" at QCIF, CIF and SD $(704 \times 576,30 \mathrm{~Hz}, 300$ frames $)$ resolutions. The following GOP structure was used: IPPP, GOP size $=16$.

Using the JM codec, we obtained the rate-distortion performance on the test video sequences, shown on Figs. 13 and 14. Using the analysis, provided in Sections 4 and 5, it is possible to evaluate the power consumption for uplink transmission taking different restrictions into account, as it was done, for example, on Figs. 8-11. Thus, we know the dependency between the power consumption and the transmission rate. Combining these results and the rate-distortion performance, results demonstrating the trade-off between video quality and power consumption can be obtained.

We used Figs. 13 and 14 and the power consumption for TSM with proposed parameters without any restrictions (Fig. 5) to obtain Figs. 15-17. Similar results can be obtained if using power consumption for TSM with proposed parameters used for Fig. 6. They illustrate that, due to the combination of the proposed TSM parameter selection with a video encoder providing constant bit rate, it is possible to adapt the level of the power consumption based on the requirements.

Fig. 13 shows the PSNR of the luminance component vs. bit rate for four standard CIF test video sequences. Fig. 14 shows the PSNR of the luminance component vs. bit rate for one test sequence

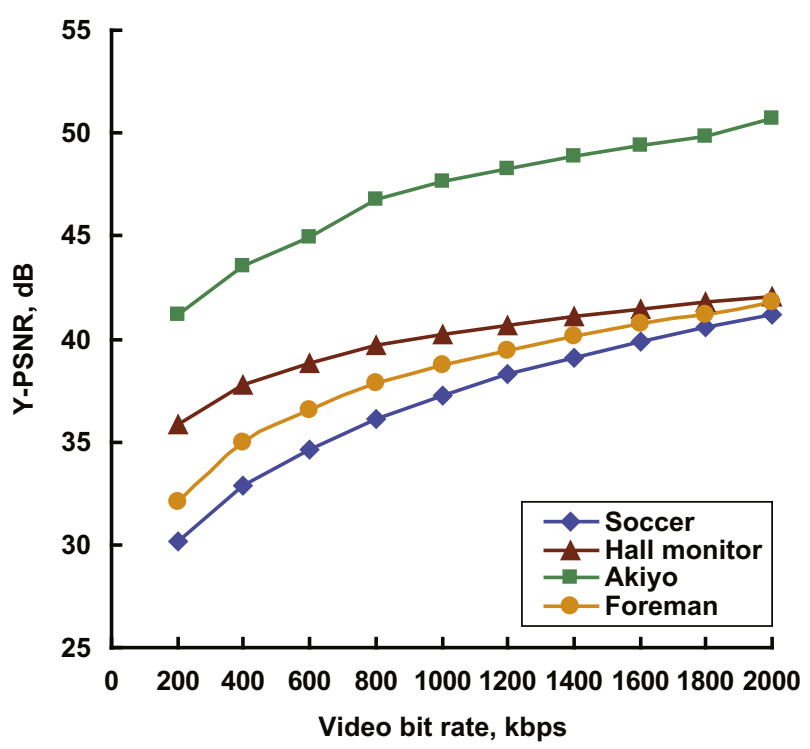

Fig. 13. Rate-distortion performance for CIF test video sequences.

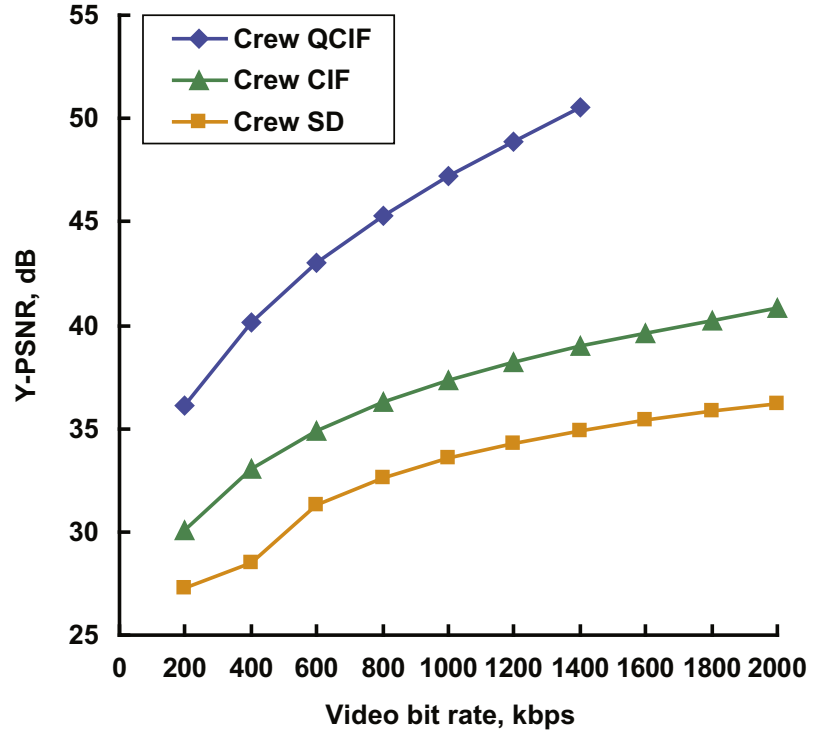

Fig. 14. Rate-distortion performance for "Crew" test video sequence.

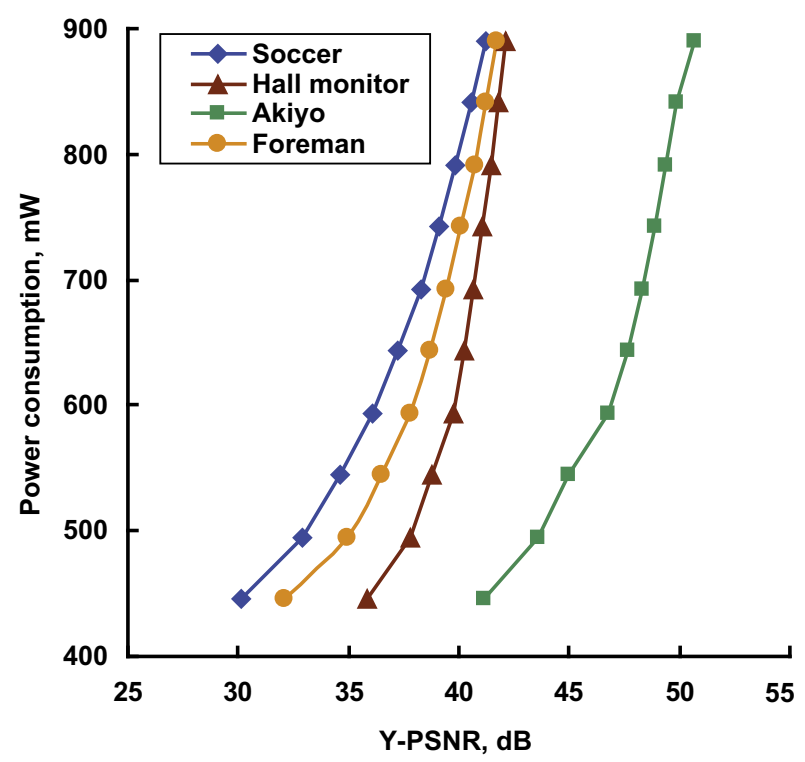

Fig. 15. Trade-off between video quality and power consumption for CIF test video sequences.

"Crew" in three different resolutions. As the content/resolution of these sequences varies (e.g. slow motion for "Akiyo" and fast motion for "Soccer", or, for example, QCIF and CIF resolutions for "Crew"), the performance of the video codec is also different. As a consequence, the same video quality for different sequences can be obtained at different bit rates.

As illustrated on Figs. 15 and 16, more power may be needed for the transmission of one sequence with a certain quality than for another sequence with the same level of quality. For example, for a quality level around $36 \mathrm{~dB}$, approximately $450 \mathrm{~mW}$ will be needed for "Hall monitor" compared to $590 \mathrm{~mW}$ for "Soccer". A similar example can be found for "Crew" sequence, when the transmission in QCIF resolution with quality around $36 \mathrm{~dB}$ will require $440 \mathrm{~mW}$ compared to approximately twice as much power for SD resolution. That means, that it is possible to control the power consumption of the UE, by defining the required level of video quality for a particular sequence. 


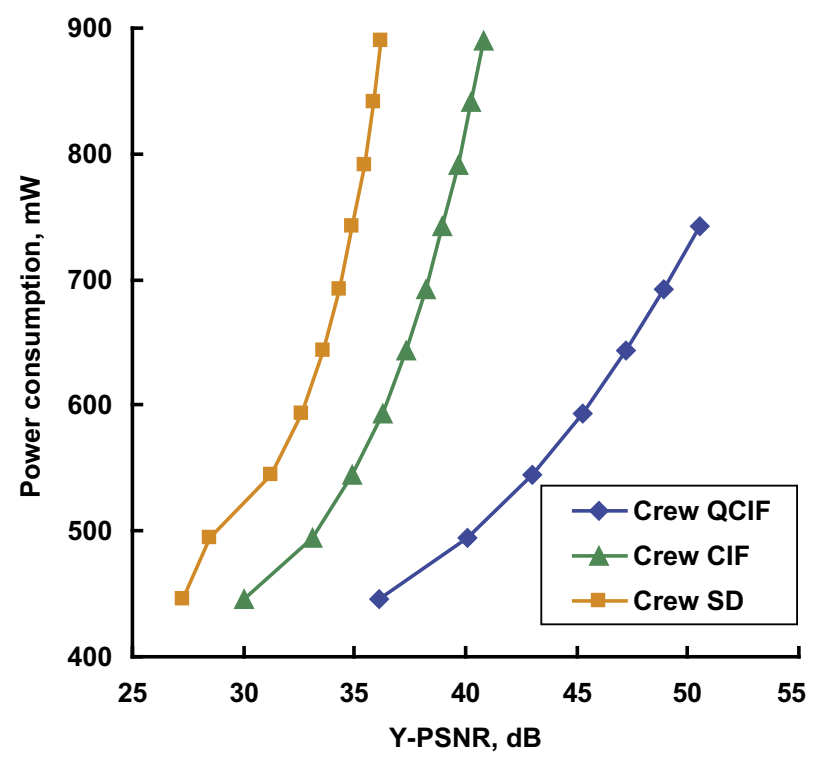

Fig. 16. Trade-off between video quality and power consumption for "Crew" test video sequence.

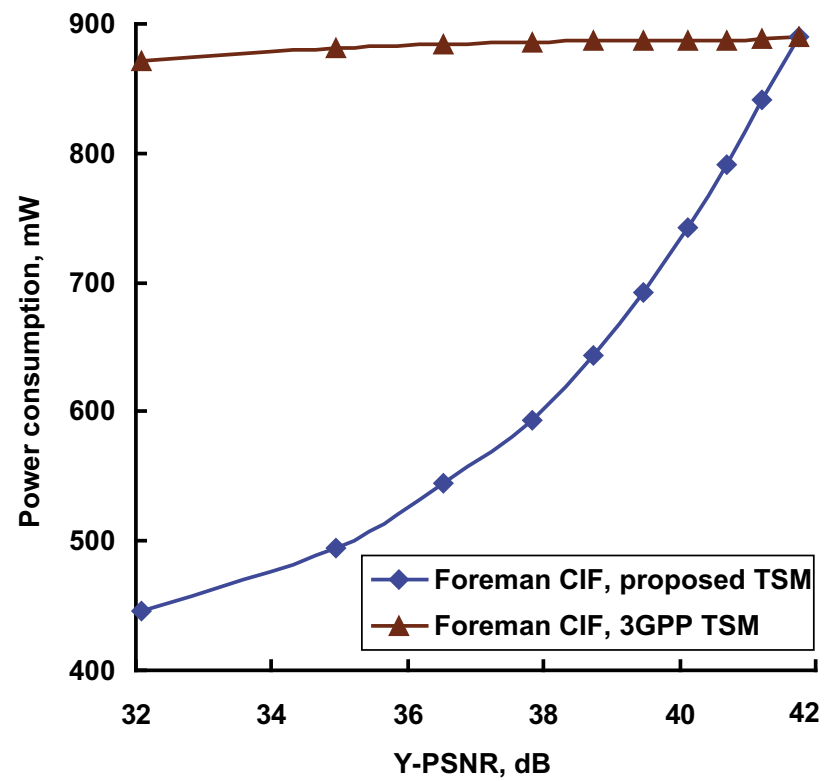

Fig. 17. Trade-off between video quality and power consumption for 3GPP and proposed transition state machines.

In fact, for some applications it would make sense to define the range of desired or acceptable quality (e.g. 30-40 dB for video transmission [42]), so that no additional power is spent for the unnecessary improvement of the video quality. Thus, for the provided data on Figs. 13 and 15, it could be beneficial to restrict the quality for "Akiyo" to say $45 \mathrm{~dB}$, and, in case of transmission of several sequences, spend the saved power on other sequences. So, we suggest that a cross-layer strategy to optimize the power and quality performances should be sequence-dependent.

As we consider the case of restricted power consumption, we try to maximize quality within these constraints. Fig. 17 shows the gain in the video quality vs. power consumption for the 3GPP and proposed TSMs for "Foreman" video sequence in CIF resolution. For the 3GPP TSM it is possible to achieve only a small gain in power consumption savings even for a significant decrease in a bit rate. The proposed state machine uses the information about the bit rate and optimizes the parameters, as was described in Section 5, so that a significant gain in power savings is achieved (up to two times for low PSNR values for the sequence "Foreman"). The proposed approach can be used for adaptive power control for the mobile device. Depending on the power resources, the transmitting device can decrease/increase the power consumption by varying the quality of the transmitted video data. For example, if it is necessary to have the high level of power savings or the transmission will last a long time, a mobile device can switch to the energy saving mode. But if there is no need for power savings on the device in the current moment, it can work with the highest quality of the video data.

\section{Conclusion}

In this paper we have considered the power consumption of data transmission over $3 \mathrm{G}$ networks for smartphones. The first part of the analysis is related to the states in RRC in general. We have proposed a power consumption model based on our experimental results on a smartphone Nokia N900, taking into account packet size and transmission intervals. Our model provides a better approximation to the experimental results than the referenced model based on data rate. The second part of the paper discussed the uplink power consumption analysis of the transition state machine. We proposed a method for parameter selection for the 3GPP transition state machine that allows to significantly decrease the uplink power consumption on the mobile device taking signaling traffic, buffer size and latency restrictions into account. Furthermore, we presented an analysis of power consumption for constant bit rate video transmission in 3G networks. We achieved a gain in power consumption vs. PSNR for transmitted video of our proposed method over the one used currently in 3GPP. Our results demonstrate that, depending on the requirements for power consumption, a mobile device can balance the video quality and transmission power, permitting to perform adaptive power management. Moreover, this work can be further extended to the LTE systems, as also there a TSM exists with a low latency for transition between idle and connected states [43]. Thus, a similar approach can be applied for energy savings in LTE.

\section{Acknowledgments}

This work was partially supported by the ECEWA project and by the Academy of Finland (Project No. 213462, Finnish Programme for Centres of Excellence in Research 2006-2011).

\section{References}

[1] The world in 2011, ICT facts and figures, International Telecommunication Union, 2011. Available on: <www.itu.int/ITU-D/ict/facts/2011/material/ ICTFactsFigures2011.pdf>

[2] T. Stockhammer, T. Oelbaum, T. Wiegand, H.26L video transmisson in 3G wireless environments, in: Proceedings of International Conference on Third Generation Wireless and Beyond (3Gwireless'02), 2002.

[3] Cisco visual networking index: global mobile data traffic forecast update, 2010-1015, 2011. Available on: <www.cisco.com>.

[4] N. Ravi, J. Scott, L. Han, L. Iftode, Context-aware battery management for mobile phones, in: Proceedings of the Sixth Annual IEEE International Conference on Pervasive Computing and Communications (PERCOM'08), 2008.

[5] G. Perrucci, Energy Saving Strategies on Mobile Devices, Ph.D. Thesis, 2009.

[6] K. Pentikousis, In search of energy-efficient mobile networking, IEEE Communication Magazine (2010).

[7] J. Zhang, D. Wu, S. Ci, H. Wang, A. Katsaggelos, Power-aware mobile multimedia: a survey, Journal of Communications 4 (9) (2009).

[8] K. Christensen, C. Gunaratne, B. Nordman, A. George, The next frontier for communications networks: power management, Computer Communications Journal 27 (2004). 
[9] J.-H. Yeh, C.-C. Lee, J.-C. Chen, Performance analysis of energy consumption in 3GPP networks, in: Proceedings of Wireless Telecommunications, Symposium, 2004.

[10] N. Sklavos, K. Touliou, A system-level analysis of power consumption and optimizations in 3G mobile devices, in: Proceedings of the 1st International Conference on NewTechnologies, Mobility and Security (NTMS'07), 2007.

[11] J. Adams, G. Muntean, Power save adaptation algorithm for multimedia streaming to mobile devices, in: Proceedings of IEEE International Conference on Portable Information Devices, 2007.

[12] J. Korhonen, Y. Wang, Power-efficient streaming for mobile terminals, in: Proceedings of the International Workshop on Network and Operating Systems Support for Digital Audio and Video (NOSSDAV'05), 2005.

[13] G. Perrucci, F. Fitzek, G. Sasso, M. Katz, Energy saving strategies for mobile devices using wake-up signals, in: Proceedings of 4th International Mobile Multimedia Communications Conference (MobiMedia 2008), 2008.

[14] B. Yu, L. Wang, J. Manner, Energy-efficient web access on mobile devices, in: Proceedings of IEEE/ACM International Conference on Green Computing and Communications \& International Conference on Cyber, Physical and Social, Computing, 2010.

[15] STMicroelectronics VS6451 (ultra small CIF+ reflowable camera module) product description. Available on: <www.st.com>.

[16] H.-C. Chang, J.-W. Chen, B.-T. Wu, C.-L. Su, J.-S. Wang, J.-I. Guo, A dynamic quality-adjustable H.264 video encoder for power-aware video applications, IEEE Transactions on Circuits and Systems for Video Technology 19 (12) (2009).

[17] Q. Zhang, Z. Ji, W. Zhu, Y. Zhang, Power-minimized bit allocation for video communication over wireless channels, IEEE Transactions on Circuits and Systems for Video Technology 12 (2002)

[18] Y. Eisenberg, C.E. Luna, T. Pappas, R. Berry, A.K. Katsaggelos, Joint source coding and transmission power management for energy efficient wireless video communications, IEEE Transactions on Circuits and Systems for Video Technology 12 (2002).

[19] Y. Chen, S. Zhang, S. Xu, G.Y. Li, Fundamental trade-offs on green wireless networks, IEEE Communications Magazine (2011).

[20] M. Kim, J. Choi, M. Kang, Trade-off guidelines for power management mechanism in the IEEE 802.16e MAC, Computer Communications Journal 31 (2008).

[21] S. De Vuyst, K. De Turck, D. Fiems, S. Wittevrongel, H. Bruneel, Delay versus energy consumption of the IEEE 802.16e sleep-mode mechanism, IEEE Transactions on Wireless Communications 8 (11) (2009).

[22] E. Belyaev, T. Koski, J. Paavola, A. Turlikov, A. Ukhanova, Adaptive power saving on the receiver side in digital video broadcasting systems based on progressive video codecs, in: Proceedings of the 11th International Symposium on Wireless Personal Multimedia, Communications, 2008.

[23] E. Belyaev, V. Grinko, A. Ukhanova, Power saving control for the mobile DVB-H receivers based on H.264/SVC standard, in: Proceedings of the 8th Wireless Telecommunication, Symposium, 2008.

[24] F. Qian, Z. Wang, A. Gerber, Z.M. Mao, S. Sen, O. Spatscheck, Characterizing radio resource allocation for $3 G$ networks, in: Proceedings of the 10th Annual Conference on Internet, Measurement, 2010.

[25] H. Haverinen, J. Siren, P. Eronen, Energy consumption of always-on applications in WCDMA networks, in: Proceedings of the 65th Semi-Annual IEEE Vehicular Technology Conference, 2007.
[26] White paper from wavion wireless networks, metro zone Wi-Fi for cellular data offloading, 2010. Available on: <www.wavionnetworks.com>.

[27] K. Mahmud, M. Inoue, H. Murakami, M. Hasegawa, H. Morikawa, Measurement and usage of power consumption parameters of wireless interfaces in energyaware multi-service mobile terminals, in: Proceedings of the 15th IEEE International Symposium on Personal, Indoor and Mobile Radio, Communications, 2004.

[28] K.-H. Lee, J.-H. Park, J.-S. Koh, User experience analysis of smartphone web surfing in UMTS networks, in: Proceedings of the 72th IEEE Vehicular Technology Conference, 2010.

[29] C. Johnson, Radio Access Networks for UMTS: Principles and Practice, John Wiley \& Sons, Ltd., 2008

[30] P.H.J. Perala, A. Barbuzzi, G. Boggia, Theory and practice of RRC state transitions in UMTS networks, in: Proceedings of 5th IEEE Broadband Wireless Access Workshop co-located with IEEE Globecom, BW-WAWS, 2009.

[31] P. Willars, Smartphone traffic impact on battery and network. Available on: $<$ www.labs.ericsson.com/developer-community/blog/smartphone-trafficimpact-battery-and-networks>.

[32] Signals research group, smartphones and a 3G network. Available on: $<$ www.signalsresearch.com>.

[33] T. Stockhammer, M.M. Hannuksela, T. Wiegand, H.264/AVC in wireless environments, IEEE Transactions on Circuits and Systems for Video Technology 13 (2003).

[34] F. Fitzek, M. Krishnam, M. Reisslein, Providing application-level QoS in 3G/4C wireless systems: a comprehensive framework based on multi-rate CDMA IEEE Wireless Communications 9 (2) (2002)

[35] N. Baghaei, R. Hunt, Review of quality of service performance in wireless LANs and $3 \mathrm{G}$ multimedia application services, Computer Communications Journal 27 (2004).

[36] MPEG-2 TM5 rate control and quantization control. Available on: http:<www.mpeg.org/MPEG/MSSG/tm5/Ch10/Ch10.html>.

[37] I. Shin, Y. Lee, H. Park, Rate control using linear rate- $\rho$ model for H.264, Signal Processing: Image Communication 19 (4) (2004).

[38] S. Sen, J.K. Dey, J.F. Kurose, J.A. Stankovic, D. Towsley, Streaming CBR transmission of VBR stored video, in: Proceedings SPIE Symposium Voice Video and Data Communications, 1997.

[39] H. Schwarz, D. Marpe, T. Wiegand, Overview of the scalable video coding extension of the H.264/AVC standard, IEEE Transactions on Circuits and Systems for Video Technology 17 (9) (2007).

[40] E. Maani, A.K. Katsaggelos, Optimized bit extraction using distortion modeling in the scalable extension of H.264/AVC, IEEE Transactions on Image Processing 18 (9) (2009).

[41] H.264/AVC JM reference software. Available on: <iphome.hhi.de/suehring/ tml>.

[42] V. Vassiliou, P. Antoniou, I. Giannakou, A. Pitsillides, Requirements for the transmission of streaming video in mobile wireless networks, in: Proceedings ICANN, 2006.

[43] Motorola, technical white paper, long term evolution (LTE): a technical overview, 2007. 